\title{
Role of CT measurement in identification of otherwise normal "dwarf" cochlea in children with sensorineural hearing loss (SNHL): Does size really matter?
}

\author{
Nikita J Nanwani, Sambhav Lodha, Nija Lingappa, Mahin Kapadia*and Shivam Batra \\ Department of Radiodiagnosis, Vadilal Sarabhai Hospital, Ahmedabad, Gujarat, India
}

\begin{abstract}
Congenital SNHL is a major health problem. Imaging findings are decision making factors in the cochlear implantation and the radiologist must be familiar with such alterations. Visual inspection alone can identify severe cochlear malformations like aplasia, common cavity deformity and incomplete partition; however visual inspection alone can miss subtle cochlear abnormalities like "dwarf" cochlea, which can be confirmed on measurements as done in our study.
\end{abstract}

\section{Introduction}

Sensorineural hearing loss (SNHL) is a major cause of disability worldwide in the paediatric population, with an estimated prevalence of 1 in 2000 neonates and 6 in 1000 children by 18 years of age. Early diagnosis and treatment of SNHL in children is critical because it improves the quality of life of these patients. Identification of inner ear malformations has direct impact on management of these children, suggesting that all children should undergo radiologic imaging as an integral component of evaluation of SNHL.

\section{Anatomy}

The inner ear comprises of the internal auditory canal, the otic capsule (surrounding the membranous labyrinth), the aqueducts and the facial nerve.

\section{Bony labyrinth}

It comprises the cochlea, vestibule and semi-circular canals. The central portion of the cavity of the bony labyrinth is called the vestibule, a large ovoid perilymphatic space measuring $4 \mathrm{~mm}$ in diameter. The vestibule is continuous anteriorly with the cochlea and posteriorly with the semi-circular canals. Each of the 3 semi-circular canals makes about $2 / 3$ rds of a circle and measures about $1 \mathrm{~mm}$ in cross-sectional diameter. Each canal is enlarged anteriorly by an ampulla while the non-ampullated ends of the superior and posterior semi-circular canals join to form the bony common crus. The cochlea is a conical structure, its base facing the IAC and its apex or cupola directed anteriorly, laterally and slightly downward. The cochlea consists of a bony canal wound around a central conical core called the modiolus. The canal winds through slightly more than $2^{1 / 2}$ turns. The first turn is the basal turn which extends into the vestibule. The thin osseous spiral lamina projects from the modiolus into the canal and partially divides it.

\section{Dwarf cochlea}

The cochlea appears relatively normal, with a normal number of turns. However, the dimensions of the cochlea are significantly smaller than normal when actually measured.

\section{Aim and objectives}

1. To establish the CT measurements of the normal cochlear height in children.

2. To evaluate the cochlear height in children with congenital SNHL who have "normal" findings at HRCT temporal bone

3. To determine radiological criteria of cochlear height correlated with SNHL.

\section{Materials and methods}

We retrospectively evaluated coronal cochlear height in 30 patients (60 ears), less than 18years of age. The study was a single blinded study carried at a tertiary care hospital in Mumbai.

\section{Control group}

- 15 patients (30 ears)

- Aged 7-18yrs(mean-14yrs)

- CT done for reasons unrelated to SNHL i.e. CSOM, Trauma, cholestetoma.

- None had undergone surgery of inner ear structures

\section{Snhl group}

- 15 patients (30 ears)

- Aged 1.5-18yrs(mean-6.4yrs)

${ }^{\star}$ Correspondence to: Mahin Kapadia, Department of Radiodiagnosis, Vadilal Sarabhai Hospital, Ahmedabad, Gujarat, India, Tel: +91 7705886633; E-mail: sambhavlodha85@hotmail.com

Key words: cochlear implantation, internal auditory canal, CT measurements

Received: December 02, 2019; Accepted: December 20, 2019; Published: December 24, 2019 
Nanwani NJ (2019) Role of CT measurement in identification of otherwise normal "dwarf” cochlea in children with sensorineural hearing loss (SNHL): Does size really matter?

- CT done before cochlear implantation

- Bilateral profound congenital SNHL

- None had CT e/o recognizable inner ear malformations

- "Normal" CT on visual inspection

\section{Imaging protocol}

The study was done on 64 slice CT Philips in a straight axial plane. The $\mathrm{kv}$ was 120 , mA 250 matrix 512 x 512 and slice thickness $0.7 \mathrm{~mm}$. The FOV was $32 \mathrm{~cm}$ and display FOV was

$9.6 \mathrm{~cm}$. The original isovolumetric data was used to obtain coronal reformatted images. The images were reviewed with high resolution bone algorithm using a small FOV separately for right and left ear. Measurement technique involved measuring the coronal height of the cochlea in strict axial and sagittal (Figure 1) planes. The measurement was done along IAC in axial plane and parallel to lateral SCC in sagittal planes.

Coronal cochlear height was measured as the maximum height including basal and upper turns taken perpendicular to the axis of cochlear lumens on reformatted coronal planes (Figure 2). The measurement was considered normal if it was within two standard deviations of the average measurements.

The above described figures explain the reference plane in which the study was done. The axial plane (Figure 3 ) and coronal plane (Figure 4) are shown for the correct measurement of the cochlear height.

\section{Statistical analysis}

It was done using SPSS 16 software, Mann Whiteney Test and Wilcoxon matched pair test.

\section{Results}

The results were interpreted using the statistical tests and presented as graphical presentation (Figures 5-9).

Dwarf cochlea incidence: cochlear height more than 2 SD $(0.30)$ below the mean (0.45) for control group. Dwarf cochlea cochlear height less than $3.9 \mathrm{~mm}$. From, the above study done, the specificity of dwarf

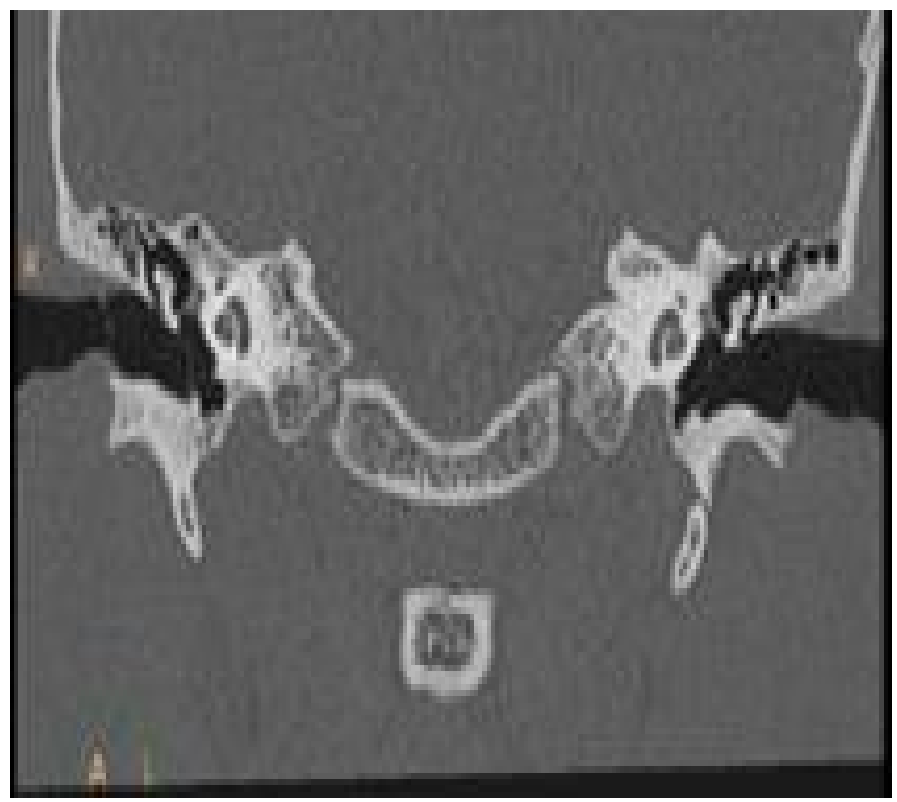

Figure 1. Coronal plane

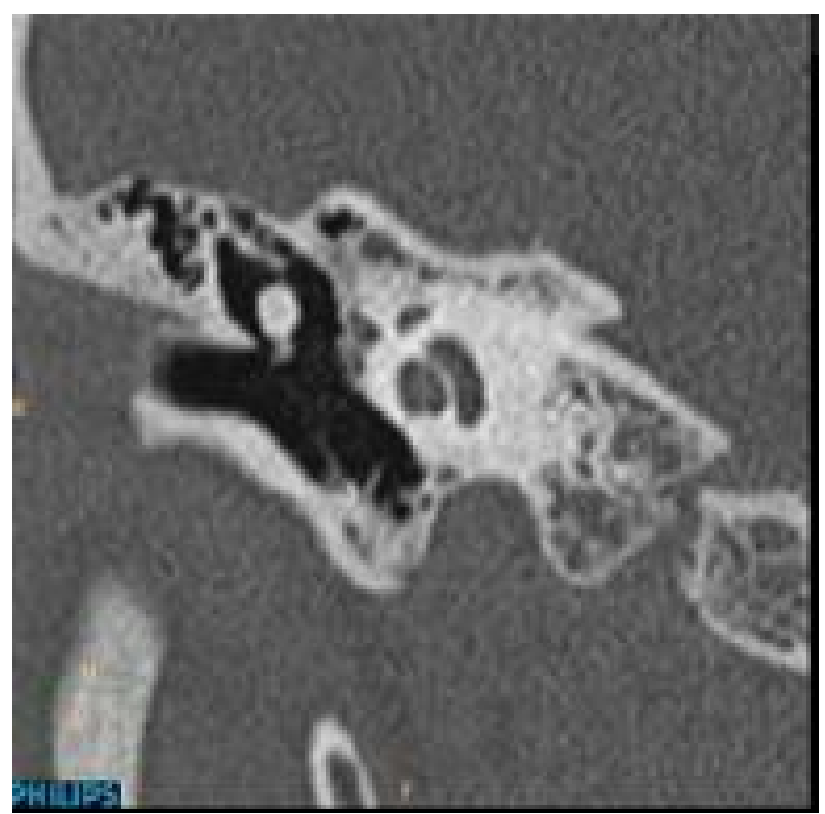

Figure 2. Saggital plane

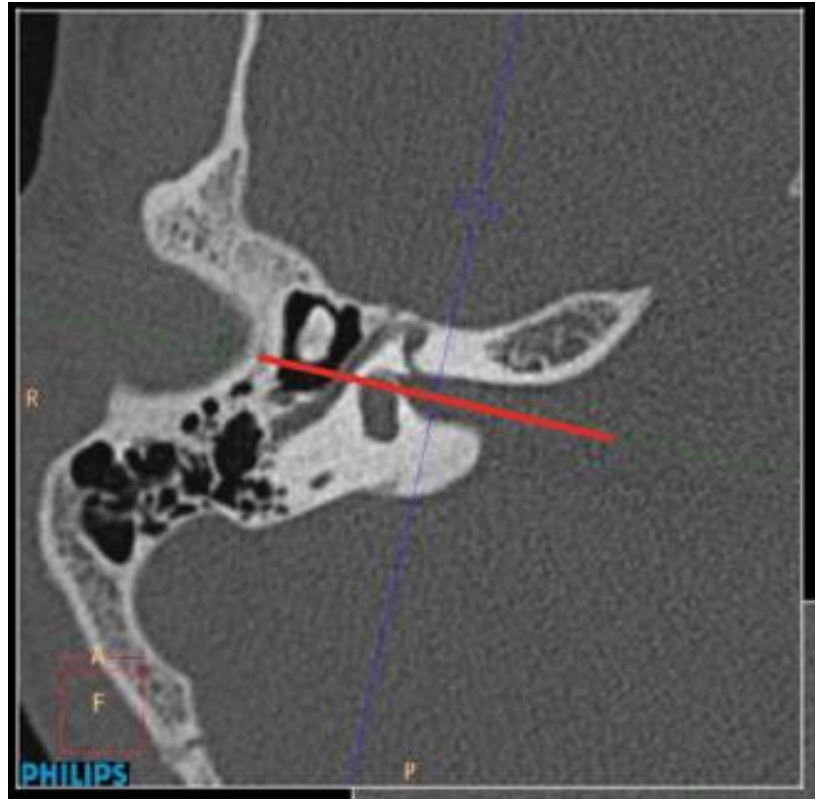

Figure 3. Axial plane

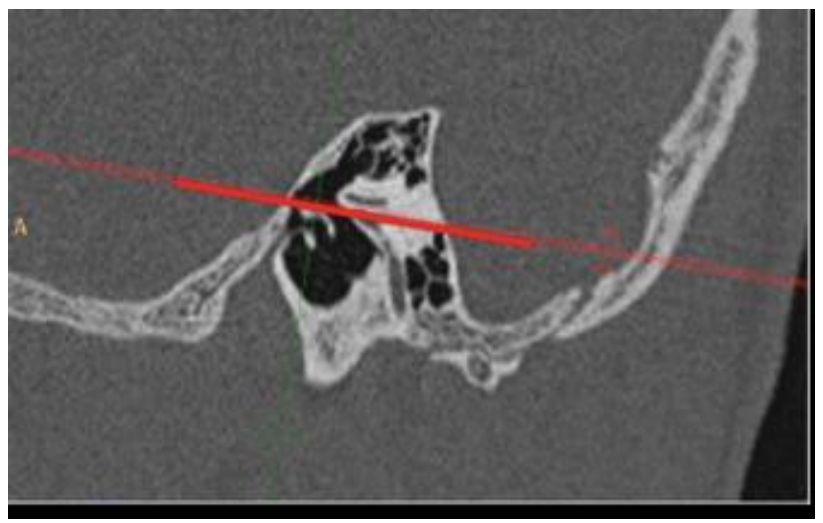

Figure 4. Coronal plane 
Nanwani NJ (2019) Role of CT measurement in identification of otherwise normal “dwarf” cochlea in children with sensorineural hearing loss (SNHL): Does size really matter?

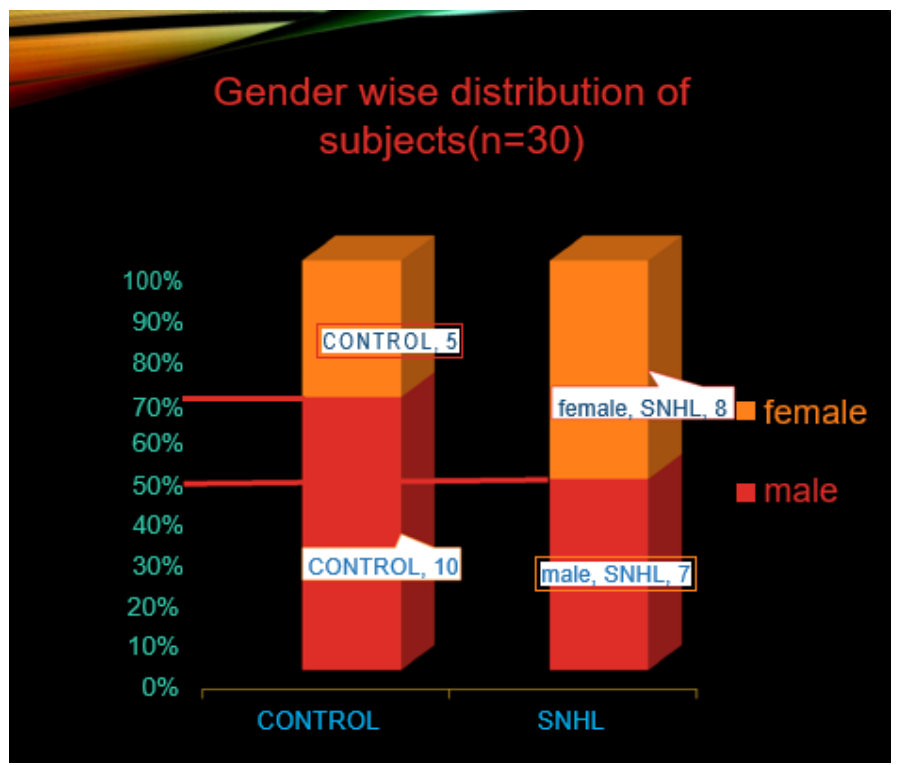

Figure 5. In the study, dwarf cochlea was observed in 10 males and 5 females from the control group whereas it was observed in 7 males and 8 females from the sensorineural hearing loss group.

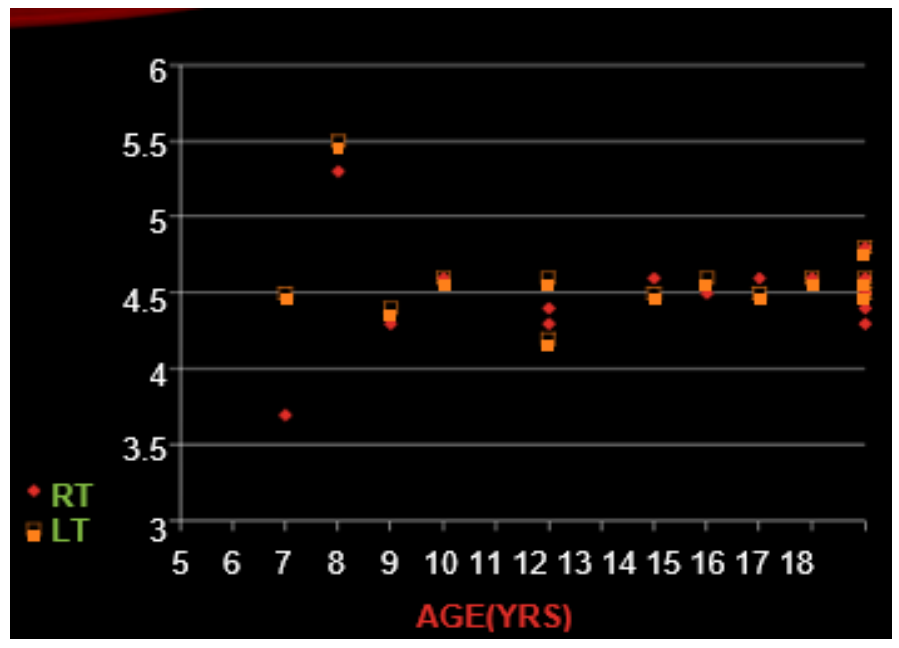

Figure 6. Comparison between cochlear height of right and left ear. The above described graph shows the comparison between right and left ears for the cochlear height, mean and SD for cochlear height for each ear in control group. The minimum cochlear height for right ear was $3.7 \mathrm{~mm}$ and maximum cochlear height was $5.3 \mathrm{~mm}$. The mean height for the right ear was 4.5 $\mathrm{mm}$ and SD value was 0.33 for right ear. The minimum cochlea height for left ear was $4.2 \mathrm{~mm}$ and maximum cochlear height was $5.5 \mathrm{~mm}$. The mean height for the left ear was 4.6 and SD value was 0.28 . The $\mathrm{p}$ value was 0.24 which finally showed no significant difference in the mean height for both the ears.

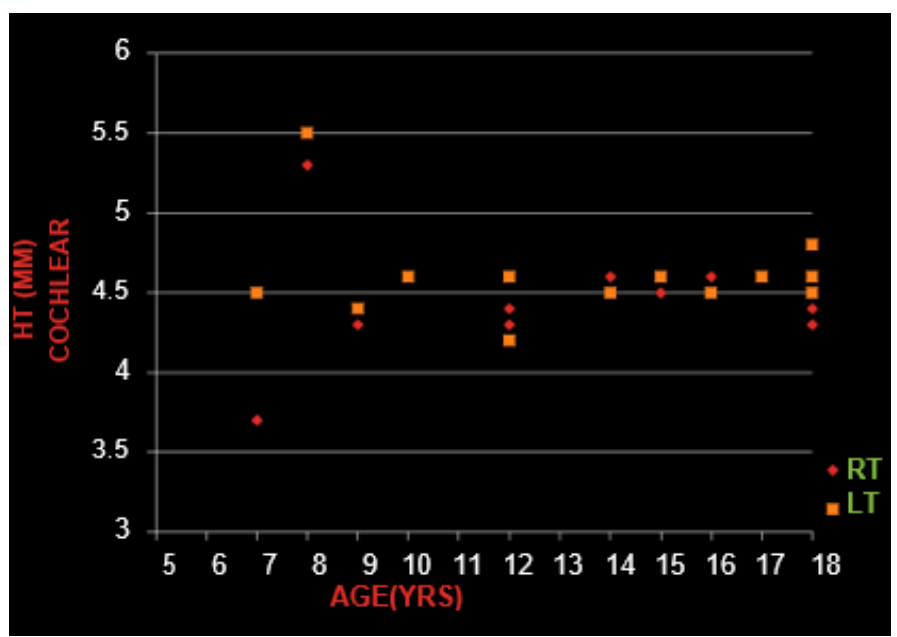

Figure 7. Influnenc of age in control group. The graph explains the influence of age in the control group. The age is taken on $\mathrm{X}$ axis and cochlear height on the $\mathrm{Y}$ axis. On applying the Pearson's correlation test, the $\mathrm{p}$ value was found to be 0.98 , which lead to the interpretation that there is no correlation between the age and cochlear height 
Nanwani NJ (2019) Role of CT measurement in identification of otherwise normal "dwarf” cochlea in children with sensorineural hearing loss (SNHL): Does size really matter?

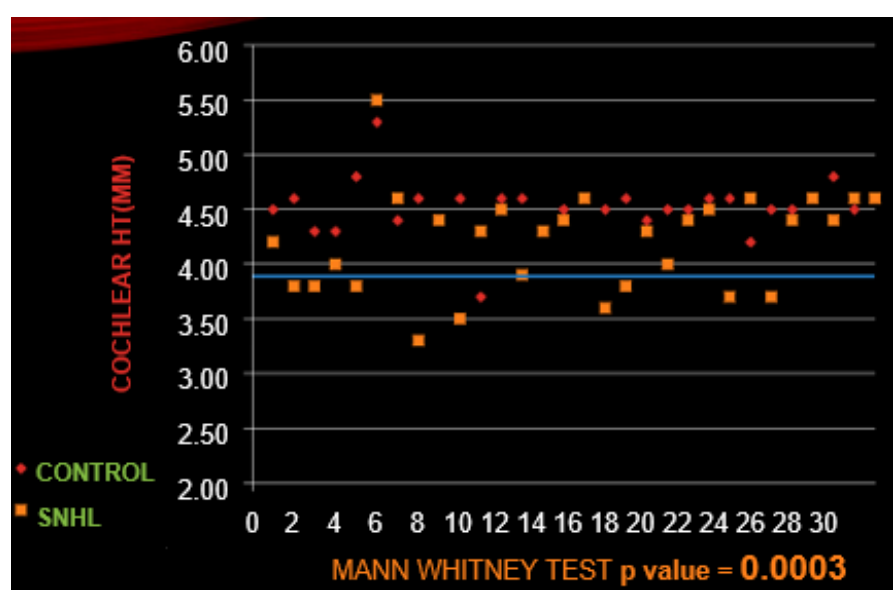

Figure 8. comparison of cochlear height between control and SNHL group. The above graph shows comparison between the cochlear height in the control and SNHL group. The mean cochlear height in the control group was $4.55 \pm 0.30$ and in SNHL group was $4.16 \pm 0.38$. The $p$ value on applying the Whiteny test was 0.0003 , which was statistically significant and showed the cochlear height was significantly smaller than control group
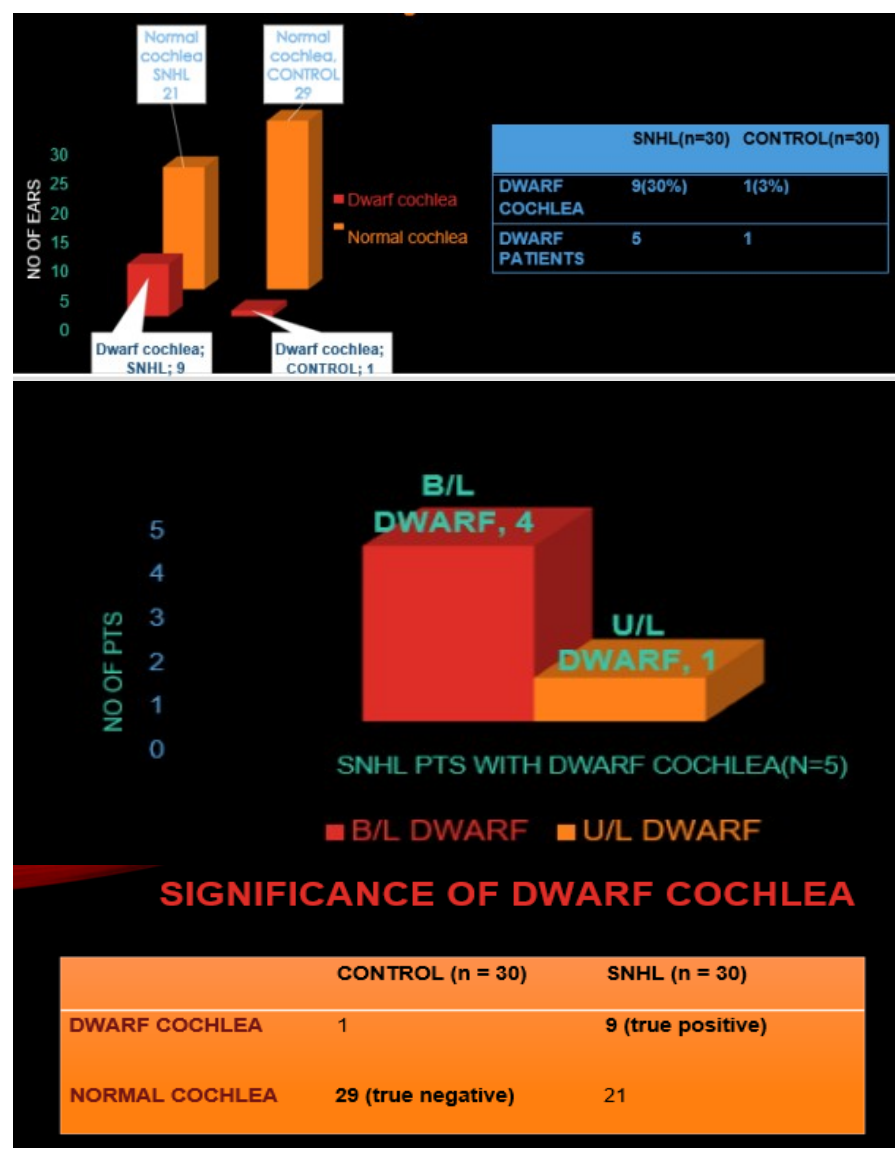

Figure 9. The bar graph explains that among the 30 patients dwarf cochlea was seen in 9 patients with SNHL and in only 1 patient in control group

cochlea in SNHL was $96 \%$, sensitivity was $30 \%$. Positive predictive value was $90 \%$.

\section{Discussion}

- HRCT is helpful in early identification and appropriate treatment of hearing loss. It is helpful in identifying the specific cause of auditory impairment. The drawback is that Visual inspection recognizes only severe malformations, but these represent mere $1 \%$ of radiographic abnormalities found in patients with SNHL.

- The most common inner ear malformations involve the vestibular aqueduct, lateral semi-circular canal, and cochlea [1].

- The major criterion for large vestibular aqueduct $(>1.5 \mathrm{~mm})$ has been previously defined [2].

- Surprisingly, similar measurements are not available for other inner ear structures.

- However, the routine measurements of different dimensions would be too cumbersome.

- Purcell et al. [3] found that coronal cochlear height was one of the few most useful measurements to diagnose common inner ear malformations.

- The coronal height is easy to identify and measure.

- We have shown the absence of variability of cochlear height with age, as has been shown in previous studies done by Mori et al. [4] Thus, normative data for cochlear height can be used independent of age.

- No significant difference between right and left ears was found. This is consistent with the results of study done by Teisser et al. [5].

- We have found significant difference in the cochlear height $(\mathrm{CH})$ between children with SNHL and without SNHL, such as described by Purcell et al. [2].

- This confirms the importance of measuring cochlear height.

- In contrast, Teisser et al. [5] did not find any significant difference in cochlear height between SNHL and non SNHL group.

- But these measurements cannot be compared to the ones obtained in our study as the reference plane used was different (axial plane).

- However, they found a significant difference when measuring cochlear width.

- Based on standardised measurement, dwarf cochlea $(\mathrm{CH}<3.9 \mathrm{~mm})$ were identified in 9 ears of SNHL that were previously missed by visual inspection.

- Dwarf cochlea was associated with SNHL with a specificity of $96 \%$ and positive predictive value of $90 \%$

- However, sensitivity was $30 \%$.

- These results are consistent with those of Purcell et al. [1] who also found cochlear hypoplasia to have high specificity and low sensitivity for SNHL.

\section{Conclusion}

$>$ Dwarf cochlea is highly predictive of SNHL

$>$ Visual inspection alone can be insensitive in the detection of subtle abnormalities like dwarf cochlea

$>$ Cochlear height, like vestibular aqueduct, should be routinely measured on all temporal bone studies.

The accurate identification of cochlear malformations will have a direct impact on the diagnosis, prognosis, and management of patients with SNHL. 
Nanwani NJ (2019) Role of CT measurement in identification of otherwise normal "dwarf” cochlea in children with sensorineural hearing loss (SNHL): Does size really matter?

\section{References}

1. Purcell D, Johnson J, Fischbein N, Lalwani AK (2003) Establishment of normative cochlear and vestibular measurements to aid in the diagnosis of inner ear malformations. Otolaryngol Head Neck Surg 128: 78-87. [Crossref]

2. Palacios E, Valvassori G (1999) Vestibular aqueduct syndrome. Ear Nose Throat J 78: 676. [Crossref]
3. Purcell DD, Fischbein NJ, Patel A, Johnson J, Lalwani AK (2006) Two temporal bone computed tomography measurements increase recognition of malformations and predict sensorineural hearing loss. Laryngoscope 116: 1439-1446. [Crossref]

4. Mori MC, Chang KW (2012) CT analysis demonstrates that cochlear height does not change with age. AJNR Am J Neuroradiol 33: 119-123. [Crossref]

5. Teissier N, Van Den Abbeele T, Sebag G, Elmaleh-Berges M (2010) Computed Tomography measurements of the normal and the pathologic cochlea in children. Pediatr Radiol 40: 275-283.

Copyright: C2019 Nanwani NJ. This is an open-access article distributed under the terms of the Creative Commons Attribution License, which permits unrestricted use, distribution, and reproduction in any medium, provided the original author and source are credited. 